\title{
Optimization of High-Quality-Factor Photonic Crystal Ring Resonator with Applications for fluid Sensing with 3D FDTD Simulation
}

\author{
R. Jannesari ${ }^{1}, T$. Grille ${ }^{2}$, B. Jakoby ${ }^{1}$ \\ 1. Institute for Microelectronics and Microsensors, Johannes Kepler University, Linz, Austria \\ 2. Infineon Technologies Austria AG, Villach, Austria \\ reyhaneh.jannesari@jku.at
}

\begin{abstract}
:
This work introduces a design and analysis for a new photonic crystal ring resonator (PCRR) for gas sensing in the mid infrared (MIR) region. By combining a photonic crystal slow light waveguide and a photonic crystal resonator, we demonstrate a new method for optical absorption sensing. For this purpose, we propose a PCRR with higher interaction between guided mode and analyte. This can be done in a PCRR consisting of dielectric silicon rods surrounded by air. We present the design and modeling of a suitable $\mathrm{PhC}$ structure utilizing silicon thus enabling fabrication in MEMS technology.
\end{abstract}

Keywords: photonic crystal ring resonator; gas sensor; resonators; integrated optics

\section{Introduction}

An optical sensor operates by measuring the changes in a property of light as it passes through an analyte. In this framework, photonic technology has been significantly enhanced fluid sensing performance. Over the past few years photonic crystal have been subject of intensive research for lab-on-chip optical technology owing to their attractive properties such as the confinement of light in a small volume, the possibility of convenient integration with waveguides and achievable ultra-high quality factors in case of resonators [1]. Photonic crystals have been proposed and their feasibility has been demonstrated for various applications including chemical and biochemical sensors [2] [3], as well as in the area of telecommunication [4]. Photonic crystal cavities can feature both, high Q-factors and small mode volume [5]. This combination together with a high filling factor (i.e. the fraction of guided light intensity interacting with the sample analyte) and low group velocity allows the enhancement of light-matter interaction, which makes these devices perfectly suitable for sensing applications. In this work, we provide a potential application for real-time detection with high sensitivity. The strategy is to simultaneously enhance the maximum field intensity and to generate the maximum field intensity of the guided mode in the region occupied by the analyte.

\section{Photonic crystal structure}

A photonic crystal $(\mathrm{PhC})$ can be described as a periodic modification of a dielectric medium featuring a period, which is in the order of the wavelength. This periodic array can be realized in a one-dimensional (1D), two-dimensional (2D) or three-dimensional (3D) fashion [6]. Using photonic crystals one can engineer dispersion properties of electromagnetic waves, propagating through it. This enables to realize strong electromagnetic interaction with an analyte for a specific wavelength $\lambda$, which is adapted to the periodicity of photonic crystal. Photonic crystals support a number of other phenomena including photonic band gap and slow light propagation. Another feature of $\mathrm{PhC}$ is the existence of local states in band gap by locally adding or removing material [7]. Ability of $\mathrm{PhCs}$ to control the flow of light, confine light in small volume, and enhance light-matter interaction makes them promising candidates for sensor applications. Researchers found that slow light (i.e. guided waves propagating with a comparatively small group velocity) can enhance light-matter interaction to realize high sensitive and miniaturized sensors [8].

In optical absorption spectroscopy, an analyte can be detected by the variation of light intensity due to absorption in analyte. The enhancement of interaction among analyte and light in the infrared range leads to enhancement of effectively detected gas absorption. 
Achieving strong confinement of light intensity in the low index region - i.e. the region filled with the analyte - is the goal of this work. This can be, e.g., obtained by utilizing a mode with low group velocity. To maximize the sensitivity, the portion of electromagnetic field associated with the mode located in the low index region has to be as large as possible. In that manner, the interaction of light and analyte, which can be a liquid or a gas, will be enhanced. The dispersion band structure of a $\mathrm{PhC}$ provides complete information about the position and the width of the band gap(s) in the relevant frequency range. In addition, the velocity and the propagation direction of electromagnetic waves in the PhC can be determined using the dispersion band diagram. The PhC parameters should be designed to create a mode whose wavelength matches the absorption peak of analyte and yields high electric field strengths in the region filled by the analyte.

\section{Photonic crystal ring resonator}

In our work, we use photonic crystal featuring hexagonal lattices. A photonic crystal ring resonator (PCRR) is created by removing elements from the regular photonic crystal grid to form a hexagonal defect. In ring resonator based devices, the choice of ring size can be adjusted to the desired resonance frequency and leads to a tradeoff between the Q-factor and mode volume. PCRRs yield several advantages including flexibility in mode design and scalability in size due to their multimode nature [9]. They can be applied as filters, lasers and sensors [10]. The proposed structure is shown in Fig.1. These types of PCRRs consist of an array of silicon rods in a hexagonal lattice, see Fig.1(a). The period and radius of the silicon rods are denoted by $a$ and $r$, respectively. The height of the silicon rods is in the order of several $\mu \mathrm{m}$. The silicon rods are residing on a silicon membrane. The lattice constant and radius of the rods is tuned to obtain a resonance peak in desired wavelength range. Fig 1(b) shows a top view of the PCRR and a waveguide to couple light into it.

The band diagram in Fig.2(a) gives the propagation modes and photonic band gap (PBG) of the photonic crystal structure, which supports quasi TE polarized modes (electric field dominantly aligned along the silicon rods). The guided modes inside PBG region are related to the PCRR cavity (red lines), where the band gap ranges from 0.2812 to $0.4526 \mathrm{a} / \lambda$. The flat PCRR modes feature a low group velocity. When the PhC slow light structure is introduced to the sample, the interaction of the light and matter will be enhanced so the absorption coefficient can be greatly increased
[8]. The Finite Difference Time Domain (FDTD) method is used to calculate the photonic band gap and propagating modes of the structure. A photonic crystal waveguide is formed by removing one line of silicon rods creating a series of defects. If the waveguide passes close to the PCRR, coupling is achieved. There are two lines of silicon rods separating the waveguide and PCRR, see Fig. 1(b). The optimum values for the height of the silicon rods and membrane are depended to the desired resonance mode. In this work, the mode we selected for sensing is the forth-created band inside the gap (M4), which is rather flat. The simulation results show that the optimum height for silicon rods and silicon membrane for M4 mode are $9 \mu \mathrm{m}$ and $0.8 \mu \mathrm{m}$, respectively. This mode is well separated from neighboring bands, which has the advantage that impinging light with specified frequency has less possibility to couple to other modes with the same frequency, which would spuriously decrease the amount of energy coupled to the desired mode.

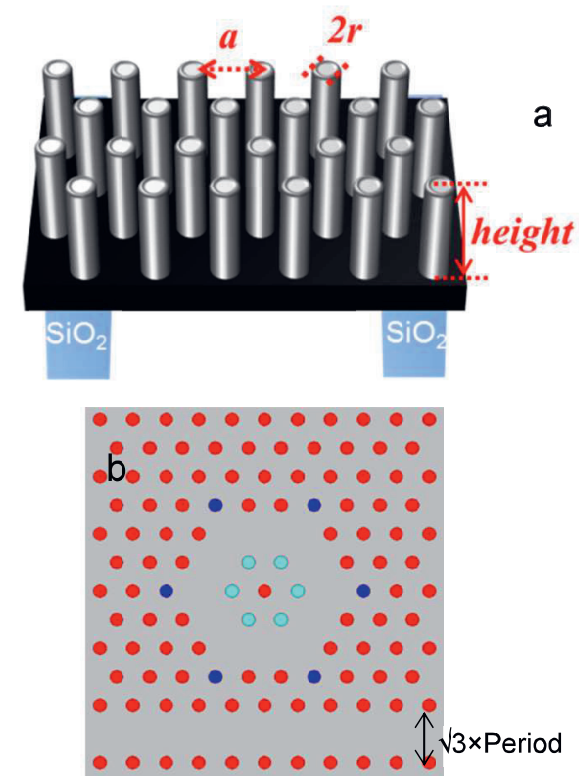

Fig.1. (a) A layout configuration of a $P C$ structure of silicon rods with $r=0.2 a$, where $r$ is radius of rods and $a$ is lattice period. (b) Proposed PCRR structure. The radius of 6 silicon rods at the corners of the outer $\mathrm{PhC}$, indicated by blue circles, and six silicon rods at the corners of inner PhC, indicate by cyan circles, is tuned to obtain higher $Q$ factor.

Achieving strong confinement of light intensity in the low index region is the goal of this work. In general, for enhance light-matter interaction phenomena there is a balance between (1) modifying the dispersion (compared to propagation through the homogeneous analyte) and (2) still maintaining a reasonable optical overlap with the analyte. In that manner, the 
interaction of light and analyte, which can be a liquid or a gas, will be enhanced. Slow light could greatly enhance light-matter interaction [11]. The theoretical basis of optical absorption is based on the Lambert-Beer law [12]. When a $\mathrm{PhC}$ slow light structure is introduced to the sample, it turns out that the interaction of the light and matter will be enhanced therefore the absorption coefficient can be enhanced by factor $\gamma=\alpha_{P h C} / \alpha \approx f c / v_{g}$.
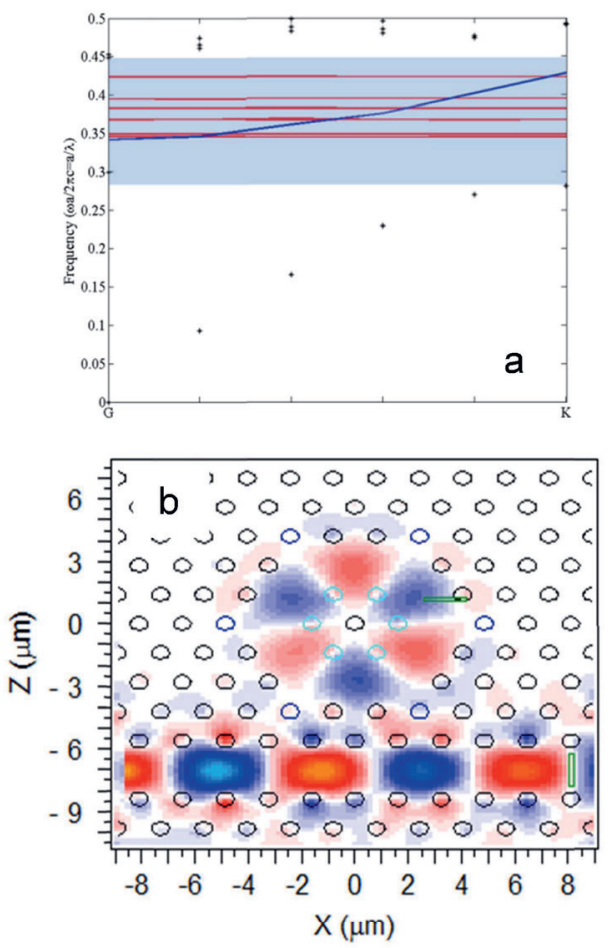

Fig. 2: (a) Band structure of the PhC (symbol *) shown together with the PCRR cavity modes (red lines) and dispersion curve of the PhC waveguide (blue line). (b) The simulated $\mathrm{E}_{\mathrm{y}}$ intensity profiles for the PCRR cavity modes

Here $\gamma$ is the dimensionless parameter of slow light enhanced absorption, $\alpha_{P h C}$ is effective absorption factor of gas in $\mathrm{PhC}, f$ is the filling factor of optical field in the analyte, which is related to the geometries of $\mathrm{PhC}, c$ is the speed of light in vacuum and $v_{g}$ is group velocity of input light in PhC [13]. The filling factor can be expressed as

$$
f \equiv \frac{E|\varepsilon| E \text { analyte }}{E|\varepsilon| E}
$$

where $\varepsilon$ is the permittivity of the analyte and $\mathrm{E}$ is the electric field of the resonant mode. The integral in the nominator of the filling factor is restricted to the region contained the analyte while the integral in the denominator extends over the entire cross section of photonic crystal. To maximize the sensitivity, the portion of electromagnetic field associated with the mode located in the low index region has to be as large as possible. Table 1 summarizes the simulated value for filling factor and normalized frequency for all six PCRR modes. The forth mode (M4) has the highest filling factor. The corresponding electric field profile confirms that the cavity mode is strongly confined within the PCRR, the overlap between electric field intensity and the fluid medium to be sensed, is 0.758 , calculated using Eq. (1). This mode has most of the electromagnetic field energy in the region of the low-index material, see Fig. 2(b), yielding the above mentioned enhanced interaction. 3D FDTD simulations show that the electric field of the mode is more confined in the middle height of the silicon rods.

In our design, the radius of the twelve silicon rods at the corners of the outer photonic crystal, indicated with blue circles, and at the corner of inner photonic crystal, indicated with cyan circles in Fig. 1(b), is tuned to obtain the higher $Q$ factor for PCRR modes in vacuum (this optimization was done in 2D). The Q-factor of forth mode (M4) rises up to more than $3.8 \times 10^{5}$ for the radii, $\mathrm{dr}_{1}=0.22 \mu \mathrm{m}$ and $d r_{2}=0.19 \mu \mathrm{m}$. In these simulations, the absorption in the silicon rods is not considered, i.e. this value for the $Q$ factor characterizes the PCRR as a non-ideal cavity resonator [14]. The Q-factor decreases by about one order of magnitude when the PCRR is coupled to waveguide. With this optimization the slow light enhanced parameter, $\gamma$, of corresponding mode goes up to the $2.127 \times 10^{4}$. In this way, the absorption is maximized and the linewidth of the absorption peak is kept narrow.

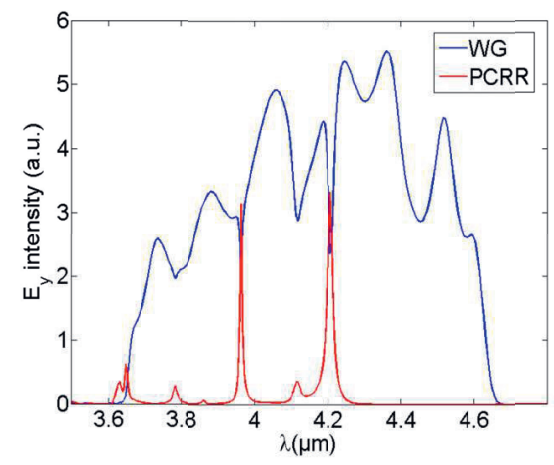

Fig.3. Simulated spectral position of the PCRR resonance modes (red curve) and transmission spectra of the PhC waveguide (blue curve)

The transmission spectra of the photonic crystal waveguide (blue curve) and spectral position of the PCRR resonance modes (red curve) are shown in Fig.3. A Gaussian input signal is launched into input port and at resonance frequency, the propagating mode is coupled from input waveguide into ring and finally to the output waveguide. The transmission efficiency is detected by monitoring the E-field intensity at 
output port of waveguide. The full 3D FDTD method was used to calculate transmission spectra of the waveguide coupled to the PCRR. The sharp dips in the transmission spectrum can be utilized to sense the absorption taking place in the PCRR, which will be the subject of future research.

\section{Conclusion}

We have presented a design for a PCRR featuring a very high $Q$ factor. $A$ simulated $Q$ factor of $3.8 \times 10^{5}$ and the slow light enhanced parameter $\gamma=5.93 \times 10^{4}$ has been achieved by slightly tuning of radius of twelve silicon rods in the inner and outer corners of the PCRR. The
PCRR is simulated by using the 3D FDTD methods. The large overlap of the high $Q$ and low group velocity photonic crystal ring resonator mode with the analyte indicates the potential for highly effective absorption sensing. The small size of the PCRR makes it suitable for parallel or multiplex detection.

\section{Acknowledgment}

The authors acknowledge the support of the Austrian COMET program (Linz Center of Mechatronics).

Tab. 1. Frequency and filling factor of PCRR modes

\begin{tabular}{|c|c|c|c|c|c|c|}
\hline Mode & M1 & M2 & M3 & M4 & M5 & M6 \\
\hline Frequency $(\mathrm{a} / \mathrm{\lambda})$ & 0.3451 & 0.349 & 0.3676 & 0.3822 & 0.395 & 0.4233 \\
\hline Filling factor & 0.585 & 0.637 & 0.658 & 0.758 & 0.71 & 0.697 \\
\hline
\end{tabular}

\section{References}

[1] M. Notomi, "Theory of light propagation in strongly modulated photonic crystals:Refractionlike behavior in the vicinity of the photonic band gap," Physical Review B, vol. 62, 15 OCTOBER 2000.

[2] Jana Jágerská, Hua Zhang, Zhaolu Diao, Nicolas Le Thomas, and Romuald Houdré, "Refractive index sensing with an air-slot photonic crystal nanocavity," Optic Letters, vol. 35, no. 15, p. 2523, 2010.

[3] Yong Zhao ,Ya-nan Zhang , Qi Wang, "High sensitivity gas sensing method based on slow light in photonic crystal waveguide," Sensors and Actuators B , vol. 173, p. 28-31, 2012.

[4] B. S. Song, S. Noda, T. Asano, "Photonic devices based on in-plane hetero photonic crystals.," Science, vol. 300, p. 1537, 2003.

[5] D. Yang, P. Zhang, H. Tian, Y.Ji, Q. Quan, "Ultrahigh-Q and Low-Mode-Volume Parabolic Radius-Modulated Single Photonic Crystal Slot Nanobeam Cavity for High-Sensitivity Refractive Index Sensing," IEEE Photonics Journal, vol. 7, no. 5, p. 4501408, 2015.

[6] J. D. Joannopoulos, S. G. Johnson, J. N. Winn, and R. D. Meade, Photonic Crystals: Molding the Flow of Light, Princeton University, 2011.

[7] E. Yablonovitch, T. J. Gmitter, R. D. Meade, A. M. Rappe, K. D. Brommer, and J. D. Joannopoulos, "Donor and acceptor modes in photonic band structure," Physical Review Letters, vol. 67, pp. 3380-3383, 1991.

[8] Yong Zhao , Ya-Nan Zhang, Qi Wang, "Research advances of photonic crystal gas and liquid sensors," Sensors and Actuators B: Chemical, vol. 160, p. 1288-1297, 2011.

[9] Se-Heon Kim, Han-Youl Ryu, Hong-Gyu Park, Guk-Hyun Kim, Yong-Seok Choi, Yong-Hee Lee, and Jeong-Soo, "Two-dimensional photonic crystal hexagonal waveguide ring laser," Applied Physics Letters, vol. 81, p. 2499, 2002.

[10] Y. Zhang, Y. Zhao , D.i Wu and Q. Wang, "Theoretical research on high sensitivity gas sensor due to slow light in slotted," Sensors and Actuators B, vol. 173, pp. 505-509, 2012.

[11] N. A. Mortensen, S. Xiao,J. Pedersen, "Liquidinfiltrated photonic crystals: enhanced lightmatter interactions for lab-on-a-chip applications," Microfluid Nanofluid, vol. 4, pp. 117-127, 2008.

[12] J. D. J.Ingle and S. R. Crouch, Spectrochemical Analysis, New Jersey: Prentice Hall, 1988.

[13] N. A. Mortensena and S. Xiao, "Slow-light enhancement of Beer-Lambert-Bouguer absorption," APPLIED PHYSICS LETTERS, vol. 90 , p. $141108,2007$.

[14] R.Jannesari, C. Ranacher, C. Consani, V. Lavchiev, T. Grille, B. Jakoby, "High-QualityFactor Photonic Crystal Ring Resonator with Applications for Gas Sensing," Procedia engineering, vol. 168, pp. 375-379, 2016. 